\title{
Pectus excavatum from a pediatric surgeon's perspective
}

\author{
Donald Nuss, Robert J. Obermeyer, Robert E. Kelly Jr \\ Department of Surgery, Eastern Virginia Medical School, 601 Children'S Lane, Norfolk, Virginia 23507, USA \\ Correspondence to: Donald Nuss, M.B., CH.B. Children'S Hospital of The King'S Daughters, Pediatric Surgery, 601 Children's Lane, Norfolk, \\ Virginia 23507, USA. Email: nussdonald@gmail.com.
}

\begin{abstract}
Historically, pectus excavatum (PE) was reported to be congenital, but in our experience only $22 \%$ are noticed in the first decade of life. Thus far, genetic studies support an autosomal recessive heritability, which coincides with only $40 \%$ of our patients having some positive family history, but is also contradictory given a constant sex ratio of 4:1 in favor of males. This inconsistency may be explained by the effect of more than one pectus disease-associated allele. Once the deformity is noticed, it tends to progress slowly until puberty, when rapid progression is often seen. We recommend surgical repair at around 12-14 years of age since the chest wall is still typically flexible and because this allows us to keep the bar in place as the patient progresses through puberty which may help decrease growth-related recurrences. Patients with mild to moderate $\mathrm{PE}$ are treated with therapeutic deep breathing, posturing, and aerobic exercises, and in appropriately selected patients, the vacuum bell may also be offered. Patients that have severe symptomatic PE are offered Minimally Invasive Repair of Pectus Excavatum (MIRPE). The surgical technique in children is similar to that of adults, except for the higher forces involved that often necessitate sternal elevation and more involved stabilization strategies. Postoperative management includes pain control, deep breathing, and early ambulation. Exercise restriction is mandatory for the first six weeks with slow resumption of normal activity after 12 weeks.
\end{abstract}

Keywords: Pediatric pectus excavatum; genetics; conservative management; minimally invasive repair; postoperative management

Submitted Mar 04, 2016. Accepted for publication May 31, 2016.

doi: 10.21037/acs.2016.06.04

View this article at: http://dx.doi.org/10.21037/acs.2016.06.04

\section{The historical perspective}

The first recorded description of pectus excavatum was in a 7 years old boy from Spain. In 1594, Bauhinus wrote "my father showed me a boy 7 years of age, among the Nobles of Andalusia, who was born with the sternum and ribs being bent back to the internal part of the chest and abdomen, so that a large cavity appeared there. It seems as though the diaphragmatic ligaments are pulling these parts inward. From birth the patient has labored with this defect, having difficulty breathing and chronic irritating cough. Now he labors very seriously, such that he seems to be in danger of suffocation because of viscid sputum. The mistress of Andalusia states that she has another grandson, much younger, born by the same daughter who is affected by the same defect (1).”
In this one paragraph, Bauhinus described all of the important features, namely, a severe pectus excavatum ("a large cavity") in a child who was acutely symptomatic ("in danger of suffocation"), that was congenital ("was born with it") and that it was genetic ("another grandson with the same defect"). Given the severity and duration of the patient's symptoms, the inevitable progression of the deformity and the fact that there were no antibiotics to treat his lung infections causes one to speculate whether he survived childhood. Unfortunately, despite this very clear description and serious nature of the condition, nothing changed for the next 350 years. It was not until the $20^{\text {th }}$ century that surgeons started to develop techniques to treat the condition.

Although several surgeons started treating children before World War II, it was Ravitch in 1949 (2) followed 
by Welch in 1958 (3) who popularized pectus excavatum repair and extended the resection from partial cartilage resection to total cartilage resection and complete mobilization of the sternum in very young children. Ravitch stated that "All the deformed cartilages are removed. The intercostal structures are transected so that the body of the sternum is free", and "Ample experience has demonstrated the ease and safety with which the operation can be performed, even in infants, and has convinced us that the younger the patient, the easier the operation for both patient and surgeon and the more likely a restitution to a normal thoracic contour. In infants, operation is advised whenever the patient is seen" (4). Welch and Shamberger stated: "The ideal age for operation is between 3 and 5 years in both boys and girls" (5).

Unfortunately, the technique of extensive rib resection and sternal isolation sometimes resulted in damage to the cartilaginous growth centers, giving rise to acquired asphyxiating chondrodystrophy. The pediatricians who followed these patients in the long-term were well aware of this problem and Sidney Gellis wrote the following in his "Pediatric Notes: The Weekly Pediatric Commentary": "It has been evident for a long time that far too many young children have had surgery for pectus excavatum. How can you judge that children less than $3 \frac{1}{2}$ years of age will have progressive disease which will permanently impair cardiac and pulmonary function (if not corrected)?" and "I have stormed for years against surgeons who have published large series of childhood patients" $(6,7)$. As a result, the pediatricians and primary care physicians became very reluctant to refer the patients for repair.

Despite the surgical popularity of the Ravitch technique for 40 years, there were a few surgeons who were not enamored with the technique and in 1990 Pena stated: "It appears necessary to develop alternative techniques that avoid the removal of costal cartilages and to re-evaluate the optimal age for repair of these malformations," but he offered no alternatives (8). In 1996, Haller and colleagues published a paper that was titled "Chest Wall Constriction After Too Extensive and Too Early Operations for Pectus Excavatum" (9). These two papers set the stage for the paradigm shift in the treatment of Pectus Exacavatum when Nuss presented his 10 year experience with the Minimally Invasive Repair of Pectus Excavatum (MIRPE) at the American Pediatric Surgery Association Congress in 1997. This was followed by the publication of the landmark MIRPE paper in the February issue of the fournal of Pediatric Surgery in 1998 (10).
The new technique changed the standard and resulted in a flood of patients seeking repair for their chest wall deformity. At The Children's Hospital of The Kings Daughters in Norfolk, Virginia, only 45 patients had a pectus excavatum repair in the decade before publication of the MIRPE technique, and in the decade after publication, the number was over 1,000 patients (11). Fonkalsrud stated: "I would particularly like to express our appreciation for stimulating the interest of parents and pediatricians in having these very common deformities come for repair, which was not the case before your report 4 years ago" (12), while Koumbourlis and Stolar stated: "the significant increase in the number of patients seeking surgical repair of the deformity during the past decade has been due to the success of the minimally invasive technique (Nuss procedure)" (13).

As other centers started performing the MIRPE procedure, numerous modifications were introduced. Three of the most significant modifications were the addition of thoracoscopy, improved bar stabilization and sternal elevation prior to tunneling across the mediastinum $(14,15)$. Secondly, with the large influx of patients, it was now possible to study cardiopulmonary function. Numerous studies have been performed since, demonstrating that there is significant cardiopulmonary dysfunction pre-operatively and significant improvement post-operatively (16-18). Research performed at The Children's Hospital of the Kings Daughters (CHKD) demonstrated a possible explanation for improved respiratory mechanics after pectus excavatum repair, using motion analysis (19). In addition, the open procedure was modified to a minimal resection technique (20).

This newly appreciated chest wall malleability also gave way for the development of non-operative therapy for pectus carinatum. Brace therapy for pectus carinatum was first reported in 1993, but in 2008 Ferro and Fraire reported their results using an innovative dynamic pressure brace. Brace therapy is reported to be successful in up to $80 \%$ of pectus carinatum patients $(21,22)$.

Finally, due to the success of the MIRPE in pre-pubertal patients, more and more teenage and adult patients have presented requesting surgery, resulting in the mean age at time of repair shifting from 6 to 15 years (Figure 1). The envelope has recently been pushed even further such that patients in their $5^{\text {th }}$ and $6^{\text {th }}$ decade are being successfully repaired (18).

\section{Congenital pectus excavatum}

In the 1996 monograph on chest wall deformities in 


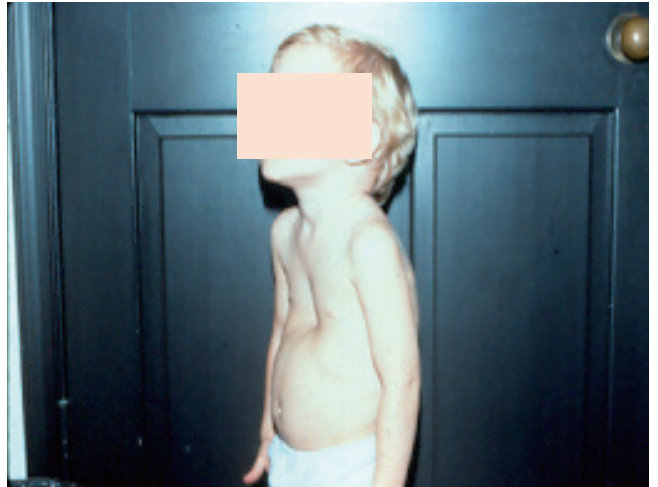

Figure 1 Eighteen month old patient with severe congenital pectus excavatum. Twenty two percent of our pectus excavatum patients are less than 10 years old.

\begin{tabular}{lll}
\multicolumn{3}{l}{ Table 1 Age of patients at time of initial presentation } \\
\hline Age (years) & Number of patients & $\begin{array}{l}\text { Percentage of } \\
\text { total sample }(\%)\end{array}$ \\
\hline$<5$ & 348 & 8.36 \\
$6-10$ & 569 & 13.67 \\
\hline $11-15$ & 1,951 & 46.88 \\
\hline $16-20$ & 957 & 22.99 \\
$>20$ & 336 & 8.07 \\
\hline Total & 4,161 & 100.0 \\
\hline
\end{tabular}

"Current Problems in Surgery", Shamberger stated: "In $90 \%$ of cases, this condition is noted within the first year of life" $(23,24)$. Our experience at CHKD is slightly different, as the age of presentation has been more common after the $10^{\text {th }}$ birthday. The patients always state that "my chest suddenly started to cave in a few months ago", and patients who present later always relate that the deformity suddenly appeared when they started their pubertal growth spurt. From the 1st of January 1987 to the 18th of November $2015,4,161$ patients were evaluated at our chest wall clinic and the age distribution is outlined in Table 1.

These numbers suggest a much lower incidence of congenital pectus excavatum than stated above. It can be reasonably assumed that the patients aged under 5 years $(8.36 \%)$ and most of the children aged under 10 (13.67\%) would have had evidence of a chest wall depression at birth, but this would still only account for $22 \%$ of the cases to be congenital.

Although the incidence of congenital pectus excavatum can be debated, the more important question is at what age should one offer surgical repair in a patient with a severe pectus excavatum. As mentioned above, from 1949 to the late 1990's, it was recommended that the younger the patient, the easier the open procedure for both the patient and surgeon. It was also suggested that the results would be superior when correction was performed at an earlier age (2-5). However, the articles by Pena and Haller, which raised the concern of subsequent osteochondrodystrophy after pre-adolescent open repair, caused the pendulum to swing in the opposite direction and most surgeons now prefer to wait until puberty or even later $(8,9)$. Since the minimally invasive repair does not require cartilage resection, it can in fact be done at any age without concern for osteochondrodystrophy. Conversely, two surgeons who have reported performing the procedure before puberty are Uemura and Park $(25,26)$. According to Uemura, reasons for this include the fact that younger patients tend to have a symmetric deformity which becomes asymmetric as the deformity progresses; they require two bars less frequently; there is less risk of bar displacement, the flexibility and symmetry of the chest in these patients makes the repair less complex; and they recover more quickly $(25,26)$. In Uemura's first series of 100 patients, $70 \%$ were less than 10 years of age with a $91 \%$ reported to have an excellent outcome. In Park's series of 1,816 consecutive patients, the mean age was 10.3 years, showing a marked preponderance of pre-pubertal patients. Long-term data from these two centers will be helpful to determine if a higher recurrence rate is appreciated later in life after the bars are removed. At CHKD, we ideally prefer to wait until the patients are 12 to 15 years of age before offering the MIRPE, since the chest wall is still malleable at that age, there is quick recovery, and recurrence rate is low since the bars will be in place past the peak age of most children's pubertal growth spurt. However, if a patient presents at a younger age with a severe deformity that qualifies for repair, we would proceed with the repair at that age.

\section{Progression of the deformity}

It is important for the surgeon to recognize that pectus excavatum is a progressive deformity during the time of growth and may progress quite rapidly during puberty. Hence, a mild deformity may become severe in less than 6 to 12 months (Figure 2). The deformity may also become more complex and more asymmetric during this time period. These morphologic changes effectively make surgical repair more difficult and this is why some surgeons 

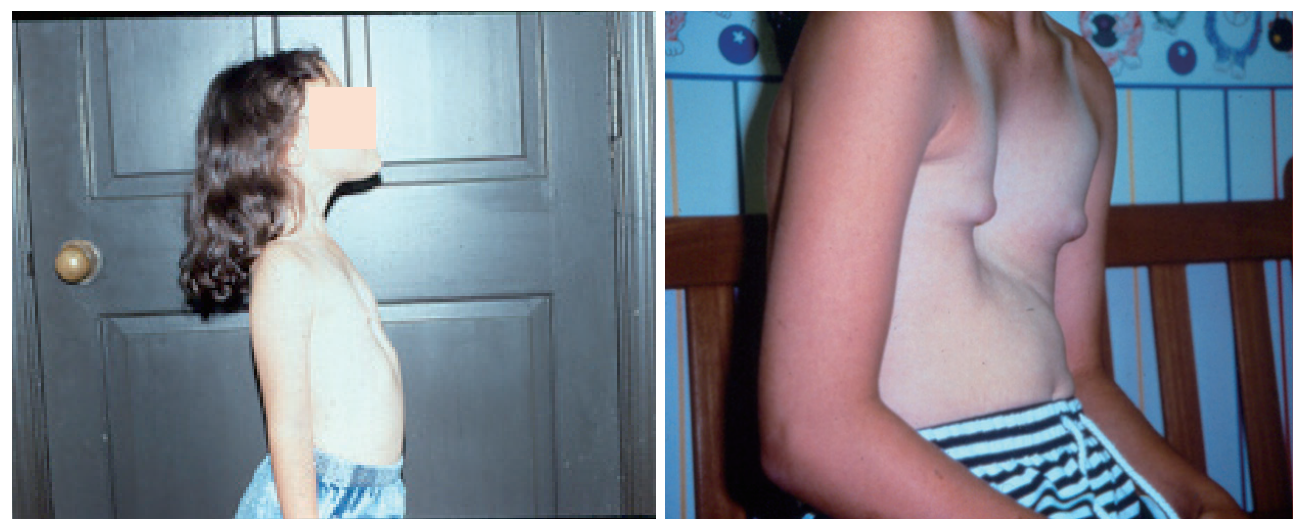

Figure 2 Pectus excavatum is a progressive deformity. This patient progressed from mild to severe \& asymmetric before puberty. At the time of puberty, the chest becomes more rigid and patients start to exhibit cardiopulmonary symptoms.

prefer to perform the MIRPE before puberty.

At CHKD, we typically follow uncorrected patients yearly, but soon after the $10^{\text {th }}$ birthday we begin to review them more frequently to help optimize the timing of surgery.

\section{Pectus excavatum genetics and associated syndromes}

Bauhinus in 1594 was the first to record a genetic trait when he described a family in which two children were born with the deformity in the same family (1). We have operated on three brothers and their two cousins all of whom had severe pectus excavatum. We have also operated on two sisters and their younger brother who all had a severe pectus excavatum. The overall incidence of familial pectus excavatum in our series is $40 \%$. Genetic studies have been ongoing without identification of a specific gene but there is strong evidence of autosomal recessive genetic control. There is likely more than one pectus disease-associated allele as well as a large number of disease allele carriers (27).

The male to female ratio has remained 4:1 in favor of boys throughout the last century. Although girls are seen less frequently, when they do come for evaluation, they usually have a more severe chest wall deformity.

Scoliosis was found to be present in just over $30 \%$ of our patients, with one-third of them being severe enough to warrant surgical correction. The other two-thirds had a mild to moderately severe scoliosis which was treated with a brace, physical therapy or observation. When confronted with a patient who has both a severe pectus excavatum and severe scoliosis, then we usually suggest that the patients' scoliosis be repaired first. If the patient is still motivated and the orthopedic surgeon is in agreement, surgical correction of their chest wall deformity will be offered 6 months later. If, on the other hand, the patients' pectus excavatum is severe enough to warrant repair while their scoliosis is mild or moderate and fails to meet the criteria for surgical correction, then we suggest proceeding with the MIRPE first.

Marfan's Syndrome and Marfanoid features have been found to be present in approximately $15 \%$ of the patients. Marfan's Syndrome is known to be associated with pectus excavatum and scoliosis in addition to aortic dilatation and lens dislocation (28). Marfan's patients tend to have more severe pectus excavatum and usually require two bars to prevent the sternum from collapsing above and below the bar. They are also prone to overcorrection, and it is therefore important that they do deep breathing and posture exercises every day in order to expand the chest and optimize their result. Overcorrection may be quite pronounced initially, but will typically improve with time.

\section{Psychological problems}

Some pectus excavatum patients, particularly those with severe defects, suffer from depression, social aversion, and poor body image. A few patients have reported suicidal ideations as a result of their deformity.

Children and teenagers have a natural aversion to deformities and therefore patients with pectus excavatum may experience severe psychological trauma both in and out of school (29). The persecution starts early as noted in the following account:

"I was 5 years old and sitting at a swimming pool learning how to swim when a boy my same age turns and 
looks at me. He points to my chest and says 'What's wrong with you?' I looked down not knowing anything was wrong with me. He looked at me strangely, got up and went to the other side of the pool. I began to hide myself as I was afraid of others seeing me. Unfortunately, one day someone at school did see me and from then on my life was torture as the boys constantly made fun of me and called me 'pigeon chest'."

\section{Conservative management}

Asymptomatic patients who are judged to have a mild or moderate deformity are treated conservatively. The conservative treatment program consists of two parts:

Firstly, the patients are started on deep breathing with breath holding exercises and a posture program. These patients are also encouraged to participate in aerobic sports activities. It is very important that the physician closely monitors the patients and that the parents take responsibility to ensure that the exercise program is properly carried out, otherwise there is almost universal failure to comply. Initially, the patients should be seen in the clinic on a monthly basis until the exercise routine is established. Involving physical therapists is beneficial since they are very skilled at working with patients who need to do regular physical therapy for a variety of musculo skeletal problems.

Secondly, patients may be offered vacuum bell therapy which requires a 1 to 2 year commitment. This requires careful instruction in the clinic and if the patient is too young to be held responsible, then the parents need to supervise the application at home. Once again, compliance with the physician directed program on a daily basis is absolutely essential for optimal results. The ideal patient for vacuum bell treatment has a mild to moderate deformity and a chest wall that is still soft and flexible. Although these patients are often less than 12 years of age, that does not rule out vacuum bell therapy for older patients. In fact, the vacuum bell may even be tried in patients with a more severe deformity and if the trial is unsuccessful, it may have helped to loosen up the anterior chest wall in preparation for surgical repair.

\section{Evaluation for possible surgery}

Our recommendation is to offer pectus excavatum repair provided the patient fulfills two or more of the following criteria:

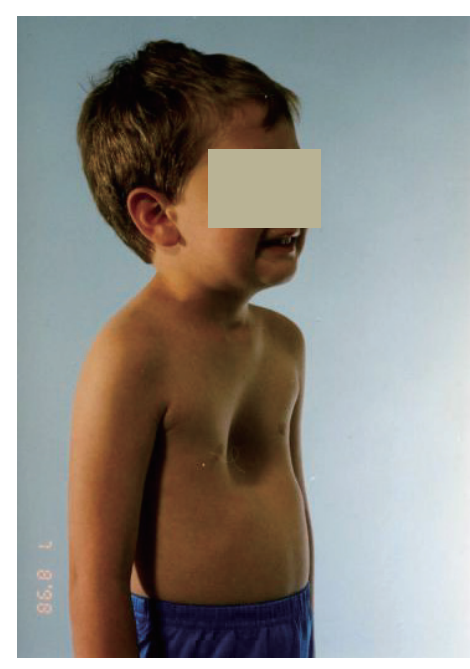

Figure 3 A congenital pectus excavatum patient who had both cardiac and pulmonary compression and fulfilled the criteria for pectus repair. He was therefore repaired at the age of 5 years.

(I) Severe and progressive deformity with associated symptoms (e.g., shortness of breath, chest pain, exercise intolerance);

(II) Chest computed tomography (CT) scan demonstrating a Haller index greater than 3.25 and showing cardiac compression or displacement;

(III) Pulmonary function studies that indicate restrictive or obstructive airway disease;

(IV) Cardiology evaluation documenting cardiac compression or displacement, mitral valve prolapse, arrhythmias, or murmurs (Figures 3,4);

(V) Previous failed open or closed repair.

\section{Minimally invasive repair (Nuss procedure)}

The operation is discussed in detail in the manuscript titled "Nuss Procedure: Past, Present and Future" in this issue of the Journal. The procedure is essentially the same for children as for adults, except that children are less likely to require two bars.

\section{Post-operative management}

The post-operative management protocols are similar for children as for older patients. Since children tend to be more anxious and less tolerant of pain, it is important to allay their anxiety before and after surgery and to preempt the pain cascade by being very pro-active with pain 

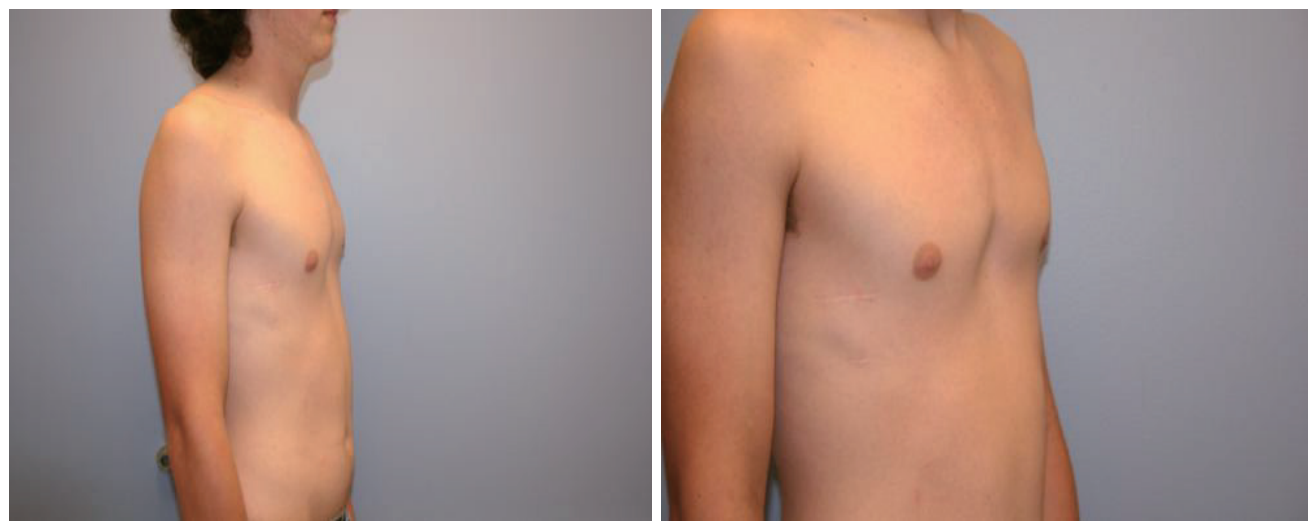

Figure 4 This is the same patient, now 15 years old and 10 years post pectus repair and 7 years post bar removal. Our philosophy is that the best age for repair is between 11 and 15 years, but we will repair whenever the patient fulfills the criteria for surgery.

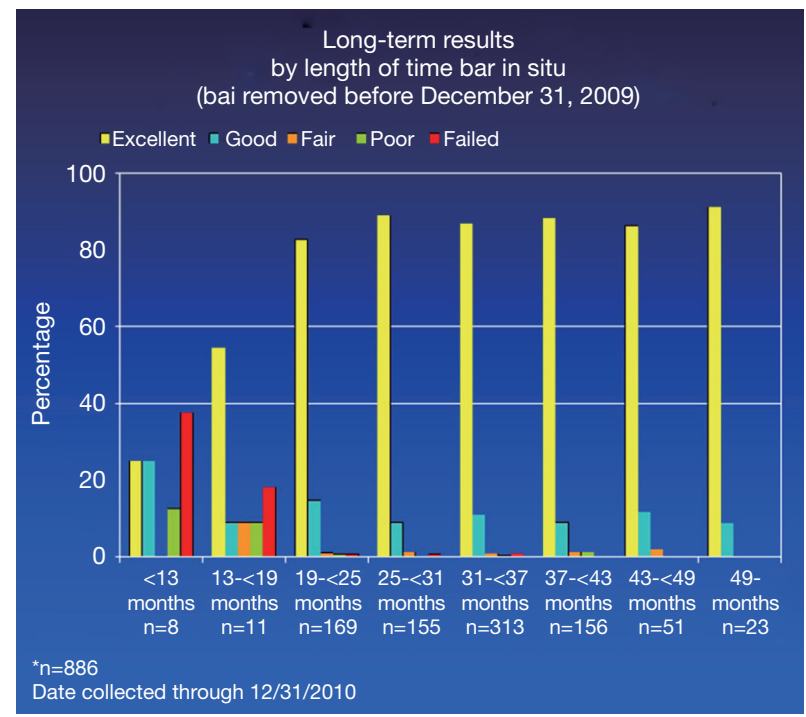

Figure 5 Graph showing the effects of different durations of bar placement.

management and sedation, especially during emergence from anesthesia and during the first 48 hours after repair. Once they have experienced breakthrough pain, they tend to become very anxious and less cooperative with breathing exercises and other activities.

On the other hand, children recover much more quickly and the patients under 12 years of age are often able to discontinue the narcotic pain medication in less than a week and discontinue all pain medications between 2 to 3 weeks, whereas adults may require narcotics for 2 to 3 weeks and NSAIDS for 4 weeks.

When patients start to feel better, it is important to remind them to refrain from sports activities, to avoid heavy lifting, and sleep on their back for a minimum of 6 weeks. After 6 weeks, they may slowly resume normal activities, and by 3 months they may resume competitive sports except for those that run the risk of significant chest wall trauma (e.g., boxing, American football, rugby, hockey, kick boxing) They should continue with deep breathing exercises twice a day until the bar has been removed and to participate in aerobic sports activities.

\section{Results}

The bar should remain in place for 2 to 4 years, but taking into consideration the severity of the chest wall deformity and the age of the patient at the time of removal for optimal timing. If the bar is removed too soon, then the recurrence rate increases as shown in Figure 5.

Ideally, the bar should remain in place for 3 years as there are no recurrences recorded after 36 months (Figure 6).

When the bar is left in place long enough, then the results of the patients less than 12 years of age are identical to the results of those over 12 years of age.

\section{Conclusions}

While the incidence of congenital pectus excavatum and heritability has not been established yet, what is currently known is that the deformity tends to become more severe as the child grows and the progression is especially significant during the pubertal growth spurt. Close observation during this time period allows the patient and family to be presented with various treatment strategies. All patients 


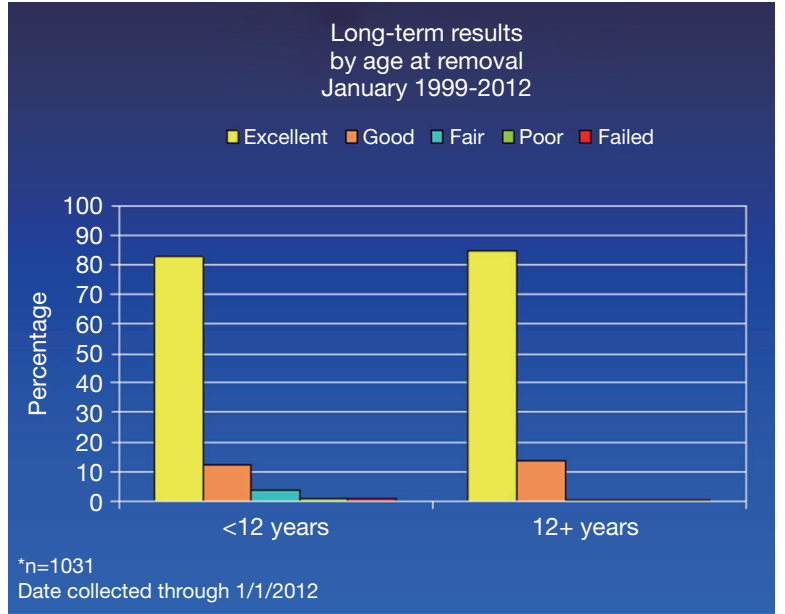

Figure 6 Shows the effect of age on long term outcome.

are started on an exercise and posture program in order to improve their posture and expand their chest, regardless of whether they are being treated operatively or nonoperatively. Vacuum bell therapy may be beneficial for a subset of patients. If the deformity is severe, as judged by specific clinical and objective criteria, then those patients should be offered MIRPE. The procedure is the same for children and adults. Postoperative protocols in the hospital and at home are very important in order to reduce the risk of complications and to improve results. The outcomes are reported as good or "excellent" by both patients and surgeons in over $98 \%$ of the cases, while less than $2 \%$ report results as "poor, fair, or failed". MIRPE is also associated with high patient satisfaction (i.e., "Very Happy" or "Happy") in over $95 \%$ of cases (28).

\section{Acknowledgements}

Thanks to Trisha Arnel, Executive Secretary.

\section{Footnote}

Conflicts of Interest: Dr. Nuss has consultation and a royalty agreement with Zimmer Biomet. Dr. Kelly and Dr. Obermeyer have consultation agreements with Biomet Zimmer Biomet.

\section{References}

1. Brown AL. Pectus Excavatum (Funnel Chest). J Thorac Surg 1939;9:164.
2. Ravitch MM. The Operative Treatment of Pectus Excavatum. Ann Surg 1949;129:429-44.

3. Welch KJ. Satisfactory surgical correction of pectus excavatum deformity in childhood; a limited opportunity. J Thorac Surg 1958;36:697-713.

4. Welch KJ, Randolph JG, Ravitch MM, et al. (eds.) Pediatric Surgery 4th Edition. Chicago: Year Book 1986;57:574.

5. Welch KJ, Shamberger RC. Chest Wall Deformities in General Thoracic Surgery, 3rd Edition, Ed. By Shields TW, 1989:515-23.

6. Gellis SS. Pediatric Notes. The Weekly Pediatric Commentary 1996;20:99-100.

7. Gellis SS. Pediatric Notes. The Weekly Pediatric Commentary 1998;22:184.

8. Calik M, Aribas OK, Kanat F. The effect of costal cartilage resection on the chest wall development: a morphometric evaluation. Eur J Cardiothorac Surg 2007;32:756-60.

9. Haller JA Jr, Colombani PM, Humphries CT, et al. Chest wall constriction after too extensive and too early operations for pectus excavatum. Ann Thorac Surg 1996;61:1618-24; discussion 1625.

10. Nuss D, Kelly RE Jr, Croitoru DP, et al. A 10-year review of a minimally invasive technique for the correction of pectus excavatum. J Pediatr Surg 1998;33:545-52.

11. Nuss D. Minimally invasive surgical repair of pectus excavatum. Semin Pediatr Surg 2008;17:209-17.

12. Croitoru DP, Kelly RE Jr, Goretsky MJ, et al. Experience and modification update for the minimally invasive Nuss technique for pectus excavatum repair in 303 patients. J Pediatr Surg 2002;37:437-45.

13. Koumbourlis AC, Stolar CJ. Lung growth and function in children and adolescents with idiopathic pectus excavatum. Pediatr Pulmonol 2004;38:339-43.

14. Park HJ, Jeong JY, Jo WM, et al. Minimally invasive repair of pectus excavatum: a novel morphology-tailored, patient-specific approach. J Thorac Cardiovasc Surg 2010;139:379-86.

15. Jaroszewski DE, Johnson K, McMahon L, et al. Sternal elevation before passing bars: a technique for improving visualization and facilitating minimally invasive pectus excavatum repair in adult patients. J Thorac Cardiovasc Surg 2014;147:1093-5.

16. Sigalet DL, Montgomery M, Harder J, et al. Long term cardiopulmonary effects of closed repair of pectus excavatum. Pediatr Surg Int 2007;23:493-7.

17. Maagaard M, Tang M, Ringgaard S,et al. Normalized 
cardiopulmonary exercise function in patients with pectus excavatum three years after operation. Ann Thorac Surg 2013;96:272-8.

18. Chao CJ, Jaroszewski DE, Kumar PN, et al. Surgical repair of pectus excavatum relieves right heart chamber compression and improves cardiac output in adult patients--an intraoperative transesophageal echocardiographic study. Am J Surg 2015;210:1118-24; discussion 1124-5.

19. Schier F, Bahr M, Klobe E. The vacuum chest wall lifter: an innovative, nonsurgical addition to the management of pectus excavatum. J Pediatr Surg 2005;40:496-500.

20. Fonkalsrud EW. Open repair of pectus excavatum with minimal cartilage resection. Ann Surg 2004;240:231-5.

21. Martinez-Ferro M, Fraire C, Bernard S. Dynamic compression system for the correction of pectus carinatum. Semin Pediatr Surg 2008;17:194-200.

22. Cohee AS, Lin JR, Frantz FW, et al. Staged management of pectus carinatum. J Pediatr Surg 2013;48:315-20.

23. Shamberger RC. Congenital chest wall deformities. Curr

Cite this article as: Nuss D, Obermeyer RJ, Kelly RE Jr. Pectus excavatum from a pediatric surgeon's perspective. Ann Cardiothorac Surg 2016;5(5):493-500. doi: 10.21037/ acs.2016.06.04
Probl Surg 1996;33:469-542.

24. Shamberger RC, Welch KJ. Surgical repair of pectus excavatum. J Pediatr Surg 1988;23:615-22.

25. Yoshida A, Uemura S, Yamamoto M, et al. Correlation of asymmetric chest wall deformity and growth in patients with pectus excavatum. J Pediatr Surg 2013;48:771-5.

26. Park HJ, Kim KS, Lee S, et al. A next-generation pectus excavatum repair technique: new devices make a difference. Ann Thorac Surg 2015;99:455-61.

27. Horth L, Stacey M, Proud VK, et al. Advancing our understanding of the inheritance and transmission of pectus excavatum. J Pediatr Genet 2012;1:161-73.

28. Kelly RE, Goretsky MJ, Obermeyer R, et al. Twentyone years of experience with minimally invasive repair of pectus excavatum by the Nuss procedure in 1215 patients. Ann Surg 2010;252:1072-81.

29. Van Horne BS, Moffitt KB, Canfield MA, et al. Maltreatment of Children Under Age 2 With Specific Birth Defects: A Population-Based Study. Pediatrics 2015;136:e1504-12. 